\title{
GALOIS CONJUGATES OF PSEUDO-ANOSOV STRETCH FACTORS ARE DENSE IN THE COMPLEX PLANE
}

\author{
BALÁZS STRENNER
}

\begin{abstract}
In this paper, we study the Galois conjugates of stretch factors of pseudo-Anosov elements of the mapping class group of a surface. We show that - except in low-complexity cases - these conjugates are dense in the complex plane. For this, we use Penner's construction of pseudo-Anosov mapping classes. As a consequence, we obtain that in a sense there is no restriction on the location of Galois conjugates of stretch factors arising from Penner's construction. This complements an earlier result of Shin and the author stating that Galois conjugates of stretch factors arising from Penner's construction may never lie on the unit circle.
\end{abstract}

\section{INTRODUCTION}

Let $S$ be a compact orientable surface. The Nielsen-Thurston classification theorem [Thu88] states that every element $f$ of the mapping class group $\operatorname{Mod}(S)$ is either finite order, reducible or pseudo-Anosov. Associated to every pseudo-Anosov element is a stretch factor $\lambda>1$ which is an algebraic integer. The goal of this paper is to study the location of Galois conjugates of pseudo-Anosov stretch factors in the complex plane.

Let $S_{g, n}$ be the orientable surface of genus $g$ with $n$ boundary components. We define the complexity of $S_{g, n}$ as $\xi\left(S_{g, n}\right)=3 g-3+n$. Note that $\xi(S)$ is half the dimension of the Teichmüller space of $S$.

The main result of the paper is the following.

Theorem 1.1. If $S$ is a compact orientable surface with $\xi(S) \geq 3$, then the Galois conjugates of stretch factors of pseudo-Anosov elements of $\operatorname{Mod}(S)$ are dense in the complex plane.

We proceed with providing motivation for the theorem. Then, at the end of the introduction, we give an outline of the proof.

Relation to Fried's problem. Every pseudo-Anosov stretch factor is a biPerron algebraic unit: an algebraic unit $\lambda>1$ whose Galois conjugates other than $\lambda$ and $1 / \lambda$ lie in the annulus $1 / \lambda<|z|<\lambda$. Fried [Fri85] asked whether or not the converse holds (up to taking powers), and it became a folklore conjecture that it does. This would give a characterization of the numbers that arise as pseudo-Anosov stretch factors. Assuming this conjecture, one

Date: January 25, 2020. 
would expect the Galois conjugates of pseudo-Anosov stretch factors to be dense in the complex plane. Theorem 1.1 is consistent with the conjecture.

Related results. Other than the bi-Perron property, little is known about the Galois conjugates of pseudo-Anosov stretch factors. Nevertheless, stretch factors of maps appear in many different but related contexts, where some results about the Galois conjugates are available.

Hamenstädt [Ham14, Theorem 1] showed that (in an appropriate sense) typical stretch factors of the homological actions of pseudo-Anosov mapping classes are totally real. If the typical pseudo-Anosov stretch factor was also totally real, then the pseudo-Anosov stretch factors we construct in this paper would be atypical, since their Galois conjugates are everywhere in the complex plane.

Thurston [Thu14] studied the stretch factors of graph maps, outer automorphisms of free groups and post-critically finite self-maps of the unit interval. He gave a characterization of such stretch factors in terms of the location of Galois conjugates: they are the so-called weak Perron numbers.

Following up on Thurston's work, Tiozzo [Tio13] studied the fractal defined as the closure of the Galois conjugates of growth rates of superattracting real quadratic polynomials and showed that this fractal is path-connected and locally connected. An analogous fractal for pseudo-Anosov stretch factors would be the closure of the Galois conjugates of pseudo-Anosov stretch factors $\lambda$ satisfying $\lambda \leq T$ for some $T>1$. As far as we know, this fractal has not yet been studied.

Construction of pseudo-Anosov mapping classes. To prove Theorem 1.1, we use the following construction of pseudo-Anosov mapping classes [Pen88] (see also [Fat92]).

Penner's Construction. Let $A=\left\{a_{1}, \ldots, a_{n}\right\}$ and $B=\left\{b_{1}, \ldots, b_{m}\right\}$ be a pair of filling ${ }^{1}$ multicurves on an orientable surface $S$. Then any product of positive Dehn twists $T_{a_{j}}$ and negative Dehn twists $T_{b_{k}}^{-1}$ is pseudo-Anosov provided that each curve is used at least once.

For each pair of multicurves $C=A \cup B$, we denote by $\mathcal{G P}(C)$ the set of Galois conjugates of stretch factors of pseudo-Anosov elements of $\operatorname{Mod}(S)$ arising from Penner's construction using the set of curves $C$. Theorem 1.1 is a corollary of the following more concrete statement.

Theorem 1.2. If $S$ is a compact orientable surface with $\xi(S) \geq 3$, then there is a collection of curves $C$ on $S$ such that $\overline{\mathcal{G P}(C)}=\mathbb{C}$.

Penner [Pen88] asked if every pseudo-Anosov mapping class has a power that arises from his construction. This was answered in the negative by Shin and the author [SS15] by showing that stretch factors arising from Penner's construction do not have Galois conjugates on the unit circle. In Question

\footnotetext{
${ }^{1}$ The components of the complement of $A$ and $B$ are disks or once-punctured disks.
} 
3.1 of the paper [SS15], we asked if such Galois conjugates can be arbitrarily close to the unit circle for a fixed collection of curves $C$. Theorem 1.2 answers this question positively.

Low complexity cases. The hypothesis on the complexity of the surface in Theorem 1.1 is necessary, because when $\xi(S) \leq 2$, the Galois conjugates of stretch factors lie on the real line and the unit circle. This is for the following reasons.

Pseudo-Anosov stretch factors arise as eigenvalues of integral symplectic matrices of size $2 \xi(S) \times 2 \xi(S)$. These matrices come from the integral piecewise linear action of the mapping class on the measured lamination space of $S$ which has dimension $2 \xi(S)$. A symplectic matrix that has an eigenvalue off the real line and the unit circle has at least 4 such eigenvalues (by complex conjugation and the fact that eigenvalues come in reciprocal pairs), so if it also has a positive real eigenvalue, its size has to be at least $6 \times 6$.

Computer experiments suggest that the Galois conjugates are dense in the real line and the unit circle when $\xi(S)=2$. However, when restricting to Penner's construction, the Galois conjugates can only be positive real. The fact that they cannot lie on the unit circle was mentioned earlier. The fact that they cannot be negative can be found in the author's thesis [Str15, Section 6.2].

Sketch of the proof. We divide the proof into three parts, corresponding to Sections 2 to 4 .

In Theorem 2.1, we give a sufficient condition for a complex number to be contained in $\mathcal{G P}(C)$ in terms of the eigenvalues of compositions of certain projections from hyperplanes to other hyperplanes in $\mathbb{R}^{n}$. This reduces to problem of approximating complex numbers by Galois conjugates of stretch factors to approximating complex numbers by eigenvalues of compositions of projections. The proof of this uses results from [Str16] stating that for certain sequences of pseudo-Anosov mapping classes arising from Penner's construction, some Galois conjugates of the stretch factors converge, and the limits are eigenvalues of a composition of projections.

In Section 3, we define the notion of rich collections of curves, and in Theorem 3.2 we show that if $C$ is a rich collection of curves, then $\overline{\mathcal{G P}(C)}=\mathbb{C}$. The main ingredient to this is showing that if $C$ is a rich collection, then every invertible linear transformation of the 2-dimensional plane can be approximated by compositions of certain projections from 2-dimensional planes to other 2-dimensional planes in $\mathbb{R}^{3}$. This allows us to apply Theorem 2.1 to conclude that all complex numbers are contained in $\overline{\mathcal{G P}(C)}$.

Finally, in Section 4 we construct rich collections of curves on various surfaces and complete the proof of Theorem 1.2.

Nonorientable surfaces. Penner's construction also works on nonorientable surfaces [Pen88, Str16], and an analog of Theorem 1.2 could be proven also for sufficiently complicated nonorientable surfaces. In the orientable case, 
we deduce Theorem 1.2 as a corollary of Theorem 3.2 and Proposition 4.1. Theorem 3.2 applies to the nonorientable case as it is, so one would only need to construct rich collections of curves on nonorientable surfaces.

\section{Galois conjugates of stretch factors in Penner's CONSTRUCTION}

The goal of this section is to establish a connection between Galois conjugates of pseudo-Anosov stretch factors and eigenvalues of certain compositions of projections.

Let $C=\left\{c_{1}, \ldots, c_{n}\right\}$ be a collection of curves used in Penner's construction. The intersection matrix $\Omega=i(C, C)$ is the $n \times n$ matrix whose $(j, k)$ entry is the geometric intersection number $i\left(c_{j}, c_{k}\right)$.

Let $Z_{i}$ be the orthogonal complement of the $i$ th row of $\Omega$. Since $\Omega$ is an intersection matrix of a collection of filling curves, all rows are nonzero and the $Z_{i}$ are hyperplanes. Let

$$
p_{i \leftarrow j}: \mathbb{R}^{n} \rightarrow Z_{i}
$$

be the - not necessarily orthogonal-projection onto the hyperplane $Z_{i}$ in the direction of $\mathbf{e}_{j}$, the $j$ th standard basis vector in $\mathbb{R}^{n}$. This projection is defined if and only if $\mathbf{e}_{j}$ is not contained in $Z_{i}$, which is in turn equivalent to the statement that the $(i, j)$-entry of $\Omega$ is positive.

Let $\mathbf{G}(\Omega)$ be the graph on the vertex set $\{1, \ldots, n\}$ where $i$ and $j$ are connected if the $(i, j)$-entry of $\Omega$ is positive. For a closed path

$$
\gamma=\left(i_{1} \cdots i_{K} i_{1}\right)
$$

in $\mathbf{G}(\Omega)$, define the linear map $f_{\gamma}: Z_{i_{1}} \rightarrow Z_{i_{1}}$ by the formula

$$
f_{\gamma}=\left.\left(p_{i_{1} \leftarrow i_{K}} \circ \cdots \circ p_{i_{2} \leftarrow i_{1}}\right)\right|_{Z_{i_{1}}} .
$$

In words, $f_{\gamma}$ is a composition of projections: first from $Z_{i_{1}}$ to $Z_{i_{2}}$, then from $Z_{i_{2}}$ to $Z_{i_{3}}$, and finally from $Z_{i_{K}}$ back to $Z_{i_{1}}$.

The following theorem gives a sufficient criterion for a complex number to be approximated by Galois conjugates of stretch factors arising from Penner's construction using a curve collection $C$.

Theorem 2.1. Let $C$ be a collection of curves satisfying the hypotheses of Penner's construction and let $\Omega=i(C, C)$. Let $\gamma$ be a closed path in $\mathbf{G}(\Omega)$ (not necessarily traversing every vertex). If $\theta$ is an eigenvalue of $f_{\gamma}$ and it is not an algebraic unit, then $\theta \in \overline{\mathcal{G P}(C)}$.

The main ingredient of the proof is a result from the paper [Str16]. Before we state the theorem, we first recall some notations from Section 2.3 of that paper.

Associated to the Dehn twists about the curves $c_{i}$ are $n \times n$ integral matrices $Q_{i}$ (depending only on $\Omega$ ) with the following property: for a product of the Dehn twists about the $c_{i}$ where every twist appears at least once, the 
corresponding product $M$ of the $Q_{i}$ is a Perron-Frobenius matrix whose leading eigenvalue equals the stretch factor of the pseudo-Anosov map.

The following is the combination of Lemma 1.2 and Theorem 3.1 of [Str16].

Theorem 2.2. Let $\Omega$ be the intersection matrix of a collection of curves satisfying the hypotheses of Penner's construction. Let $\gamma=\left(i_{1} \ldots i_{K} i_{1}\right)$ be a closed path in $\mathbf{G}(\Omega)$ visiting each vertex at least once. Let

$$
M_{\gamma, k}=Q_{i_{K}}^{k} \cdots Q_{i_{1}}^{k}
$$

and let $\lambda_{k}$ be the Perron-Frobenius eigenvalue of $M_{\gamma, k}$. Denote by $u_{k}(x)$ and $v(x)$ the characteristic polynomials $\chi\left(M_{\gamma, k}\right)$ and $\chi\left(f_{\gamma}\right)$, respectively. Then we have

$$
\frac{u_{k}(x)}{x-\lambda_{k}} \rightarrow v(x)
$$

If, in addition, $v(\theta)=0$ and $\theta_{k} \rightarrow \theta$ is a sequence such that $u_{k}\left(\theta_{k}\right)=0$ and $\theta_{k} \neq \theta$ for all but finitely many $k$, then $\theta_{k}$ and $\lambda_{k}$ are Galois conjugates for all but finitely many $k$.

We are now ready to prove Theorem 2.1.

Proof of Theorem 2.1. Since $\chi\left(f_{\gamma}\right)$ is invariant under homotopy of $\gamma$ [Str16, Proposition 4.1] and the graph $\mathbf{G}(\Omega)$ is connected, we may assume that $\gamma=\left(i_{1} \ldots i_{K} i_{1}\right)$ traverses every vertex. Then each matrix $M_{\gamma, k}$ corresponds to a pseudo-Anosov mapping class with stretch factor $\lambda_{k}$.

The characteristic polynomials $u_{k}(x)$ of $M_{\gamma, k}$ are monic and have constant coefficient \pm 1 , because the matrices $Q_{i}$ are invertible. This can be seen directly from the definition of the matrices $Q_{i}$ in Section 2.3 of [Str16]. So the roots of $u_{k}(x)$ are algebraic units.

By the first part of Theorem 2.2, there is sequence $\theta_{k} \rightarrow \theta$ such that $u\left(\theta_{k}\right)=0$ for all $k$. Since $\theta$ is assumed not to be an algebraic unit, we have $\theta_{k} \neq \theta$ for all but finitely many $k$. By the second part of Theorem 2.2, this implies that $\theta_{k}$ is a Galois conjugate of $\lambda_{k}$. So the number $\theta$ is indeed approximated by Galois conjugates of Penner stretch factors arising from the collection $C$.

\section{Approximation of linear maps by COMPOSITIONS OF PROJECTIONS}

In this section we define rich collections of curves and prove that if $C$ is such a collection of curves, then we have $\overline{\mathcal{G P}(C)}=\mathbb{C}$. This will reduce our main theorem to the problem of constructing rich collections of curves on various surfaces. First, we need the following definitions.

We define the cross-ratio of a $2 \times 2$ matrix

$$
M=\left(\begin{array}{ll}
m_{11} & m_{12} \\
m_{21} & m_{22}
\end{array}\right)
$$

by the formula $\operatorname{cr}(M)=\frac{m_{11} m_{22}}{m_{12} m_{21}}$. In order for the cross-ratio to be defined, all matrices are assumed to have positive entries throughout this section. 
Denote by $\mathbb{R}_{+}^{\times}$the multiplicative group of the positive reals. The crossratio group $\operatorname{CRG}(M)$ of a matrix $M$ is the subgroup of $\mathbb{R}_{+}^{\times}$generated by the cross-ratios of all $2 \times 2$ submatrices of $M$. Note that any subgroup of $\mathbb{R}_{+}^{\times}$is either trivial, infinite cyclic or dense.

Definition 3.1. We call a collection of curves $C=\left\{c_{1}, \ldots, c_{n}\right\}$ on a surface $S$ rich if

- $C$ fills $S$,

- $n \geq 6$ and $C_{1}=\left\{c_{1}, c_{2}, c_{3}\right\}$ and $C_{2}=\left\{c_{4}, c_{5}, c_{6}\right\}$ form multicurves,

- $i\left(C_{1}, C_{2}\right)$ has positive entries, rank 3 and dense cross-ratio group.

Theorem 3.2 (Criterion for density of Galois conjugates). If $C$ is a rich collection of curves on $S$, then $\overline{\mathcal{G P}(C)}=\mathbb{C}$.

The following subsections develop material necessary for the proof. The proof will be given at the end of the section.

3.1. Bipartite subgraphs. Suppose $1 \leq k_{1}<k_{2} \leq n$ and let

$$
\begin{aligned}
& I=\left\{1, \ldots, k_{1}\right\} \\
& J=\left\{k_{1}+1, \ldots, k_{2}\right\} .
\end{aligned}
$$

Suppose that $\omega_{i j}=0$ whenever $i, j \in I$ or $i, j \in J$, and $\omega_{i j}>0$ when $i \in I, j \in J$ or $j \in I, i \in J$. In other words, we assume that the subgraph of $\mathbf{G}(\Omega)$ spanned by the vertices $I \cup J$ is a complete bipartite graph. Compare the setting $I=\{1,2,3\}$ and $J=\{4,5,6\}$ with the definition of rich collections of curves.

Define the subspace

$$
V=\left\langle\mathbf{e}_{k_{1}+1}, \ldots, \mathbf{e}_{k_{2}}\right\rangle
$$

generated by the standard basis vectors indexed by $J$. For $1 \leq i \leq k_{1}$, the subspace

$$
X_{i}=V \cap Z_{i}
$$

is a hyperplane in $V$, because $Z_{i}$ is hyperplane in $\mathbb{R}^{n}$ and $V \not \subset Z_{i}$. When $i \in I$ and $j \in J$, the projection $p_{i \leftarrow j}$ restricts to $V$ and induces a projection

$$
s_{i \leftarrow j}: V \rightarrow X_{i}
$$

in the direction of $\mathbf{e}_{j}$. On the other hand, the restriction $s_{j \leftarrow i}$ of $p_{j \leftarrow i}$ on $V$ is the identity.

Let $\gamma=\left(i_{1} j_{1} \ldots i_{K} j_{K} i_{1}\right)$ be a closed path in $\mathbf{G}(\Omega)$, starting at $i_{1} \in I$, and assume that it only traverses the vertices in $I \cup J$. Then $f_{\gamma}$ induces a linear endomorphism $s_{\gamma}: X_{i_{1}} \rightarrow X_{i_{1}}$ by the formula

$$
s_{\gamma}=\left.\left(s_{i_{1} \leftarrow j_{K}} \circ s_{j_{K} \leftarrow i_{K}} \circ \cdots \circ s_{i_{2} \leftarrow j_{1}} \circ s_{j_{1} \leftarrow i_{1}}\right)\right|_{X_{i_{1}}},
$$

which simplifies to

$$
s_{\gamma}=\left.\left(s_{i_{1} \leftarrow j_{K}} \circ \cdots \circ s_{i_{3} \leftarrow j_{2}} \circ s_{i_{2} \leftarrow j_{1}}\right)\right|_{X_{i_{1}}},
$$

since the omitted terms are the identity maps. Since $s_{\gamma}$ is simply the restriction of $f_{\gamma}$ to the invariant subspace $X_{i_{1}}$, we have the following. 
Proposition 3.3. The characteristic polynomial of $s_{\gamma}$ divides the characteristic polynomial of $f_{\gamma}$.

In other words, the eigenvalues of $s_{\gamma}$ form a subset of the eigenvalues of $f_{\gamma}$. Thus having control over the eigenvalues of $s_{\gamma}$ is useful for applying Theorem 2.1.

In the proof of [Str16, Proposition 4.1], it was shown that $f_{\gamma}$ is invariant under homotopies of $\gamma$ that fix the last edge of $\gamma$. This property is inherited by $s_{\gamma}$. In fact a stronger homotopy invariance holds for $s_{\gamma}$ : it is invariant under all homotopies fixing the base point $i_{1}$, without the assumption that the last edge of $\gamma$ is fixed throughout the homotopy. To see this we only need to check that the removal of the backtracking $i_{K} j_{K} i_{1}$, when $i_{K}=i_{1}$, from $\gamma$ does not change $s_{\gamma}$. This is because only the projection $\left.s_{i_{1} \leftarrow j_{K}}\right|_{X_{i_{K}}}$ is dropped from the composition, but it is a projection from $X_{i_{1}}$ to $X_{i_{1}}$ so it does not have any effect.

As a consequence, the map $\gamma \mapsto s_{\gamma}$ induces a well-defined map

$$
\rho_{i_{1}}: \pi_{1}\left(G^{\prime}, i_{1}\right) \rightarrow \operatorname{End}\left(X_{i_{1}}\right)
$$

where $G^{\prime}$ is the subgraph of $\mathbf{G}(\Omega)$ spanned by the vertex set $I \cup J$ and $\operatorname{End}\left(X_{i_{1}}\right)$ is the set of linear endomorphisms of $X_{i_{1}}$. Moreover, this map is an anti-homomorphism:

$$
s_{\gamma_{1} * \gamma_{2}}=s_{\gamma_{2}} \circ s_{\gamma_{1}} .
$$

This property reduces the computation of $s_{\gamma}$ for a long path $\gamma$ to the computation of $s_{\gamma}$ for short paths $\gamma$. It also shows that the image of $\rho_{i_{1}}$ is in fact in $\mathrm{GL}\left(X_{i_{1}}\right)$.

3.2. An example. Let $I=1,2$ and $J=3,4$. The upper left $4 \times 4$ submatrix of $\Omega$ has the block form $\left(\begin{array}{cc}0 & Y \\ Y^{T} & 0\end{array}\right)$, where $Y=\left(\begin{array}{ll}a & b \\ c & d\end{array}\right)$ is a $2 \times 2$ matrix with positive entries. Now $V$ is the 2-dimensional subspace generated by $\mathbf{e}_{3}$ and $\mathbf{e}_{4}$. The hyperplanes $Z_{1}$ and $Z_{2}$ are the orthogonal complements of first and second rows of $\Omega$. Hence $X_{1}$ and $X_{2}$ are the lines in $V$ with equations $a \mathbf{e}_{3}+b \mathbf{e}_{4}=0$ and $c \mathbf{e}_{3}+d \mathbf{e}_{4}=0$. The slopes are $-\frac{b}{a}$ and $-\frac{d}{c}$, respectively. The lines are illustrated on Figure 3.1.

Let $\gamma$ be some non-contractible closed path of length 4 in the subgraph $G^{\prime}$ of $\mathbf{G}(\Omega)$ spanned by $I \cup J$. For instance, let $\gamma=(14231)$. Then $s_{\gamma}=$ $\left.\left.s_{1 \leftarrow 3}\right|_{X_{2}} \circ s_{2 \leftarrow 4}\right|_{X_{1}}$. The projection $\left.s_{2 \leftarrow 4}\right|_{X_{1}}: X_{1} \rightarrow X_{2}$ changes only the $\mathbf{e}_{4}$-coordinate of points, and it changes it by a factor $\frac{a d}{b c}$ which is the ratio of the slopes of $X_{2}$ and $X_{1}$. The projection $\left.s_{1 \leftarrow 3}\right|_{X_{2}}$ then projects back onto $X_{1}$ without changing the $\mathbf{e}_{4}$-coordinate. Hence the composition is a scaling of the line $X_{1}$ by a factor $\frac{a d}{b c}$. Therefore $\frac{a d}{b c}$ is an eigenvalue of $s_{\gamma}$ and $f_{\gamma}$.

The graph $G^{\prime}$ is topologically a circle and $\rho_{1}: \pi_{1}\left(G^{\prime}, 1\right) \rightarrow \operatorname{End}\left(X_{1}\right)$ maps the generator of the infinite cyclic group to the scaling of $X_{1}$ by $\frac{a d}{b c}$. As a consequence, the eigenvalues of $s_{\gamma}$ for closed paths $\gamma$ in $G^{\prime}$ are precisely the integer powers of $\frac{a d}{b c}$. 


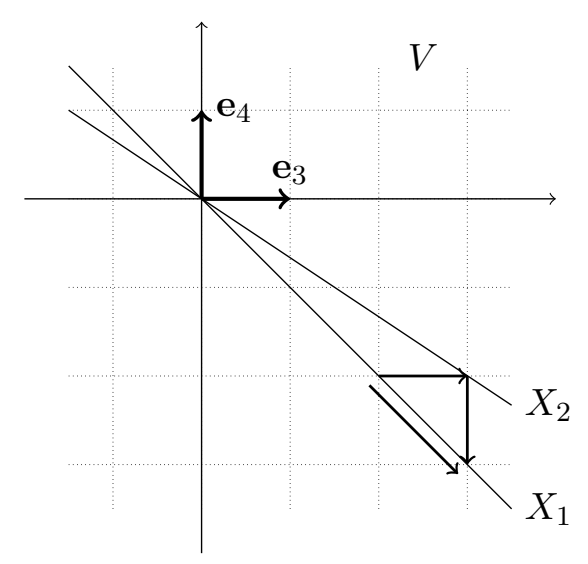

Figure 3.1. The map $s_{\gamma}: X_{1} \rightarrow X_{1}$.

3.3. Short closed paths. The content of this section is a geometric description of $s_{\gamma}$ when $\gamma$ is a path of length 4. Section 3.2 discussed the simple special case when $G^{\prime}$ is a graph on four vertices. In this section we allow $G^{\prime}$ to be bigger, hence $V$ to have dimension greater than 2 .

Consider a closed path $\left(i_{1} j_{1} i_{2} j_{2} i_{1}\right)$ where $i_{1}, i_{2} \in I$ and $j_{1}, j_{2} \in J$. Associated to $\left(i_{1} j_{1} i_{2} j_{2} i_{1}\right)$ are the following data:

$$
\begin{aligned}
& \text { - } \Omega_{i_{1} i_{2}}^{j_{1} j_{2}}=\left(\begin{array}{ll}
\omega_{i_{1} j_{1}} & \omega_{i_{1} j_{2}} \\
\omega_{i_{2} j_{1}} & \omega_{i_{2} j_{2}}
\end{array}\right), \\
& \text { - } c_{i_{1} j_{2}}^{j_{1} j_{2}}=\operatorname{cr}\left(\Omega_{i_{1} i_{2}}^{j_{1} j_{2}}\right), \text { and } \\
& \text { - } s_{i_{1} j_{2}}^{j_{1} i_{2}}=s_{\left(i_{1} j_{1} i_{2} j_{2} i_{1}\right)}=\left.\left.s_{i_{1} \leftarrow j_{2}}\right|_{X_{i_{2}}} \circ s_{i_{2} \leftarrow j_{1}}\right|_{X_{i_{1}}} \in \operatorname{GL}\left(X_{i_{1}}\right) .
\end{aligned}
$$

Note that $\left(s_{i_{1} i_{2}}^{j_{1} j_{2}}\right)^{-1}=s_{i_{1} i_{2}}^{j_{2} j_{1}}$, since $\left(i_{1} j_{1} i_{2} j_{2} i_{1}\right)$ and $\left(i_{1} j_{2} i_{2} j_{1} i_{1}\right)$ represent inverse elements in $\pi_{1}\left(G^{\prime}, i_{1}\right)$. So the monoid generated by the linear maps $s_{i_{1} i_{2}}^{j_{1} j_{2}} \in \mathrm{GL}\left(X_{i_{1}}\right)$, where $\left(i_{1} j_{1} i_{2} j_{2} i_{1}\right)$ runs through all closed paths of length 4 in $G^{\prime}$ with base point $i_{1}$, is actually a group.

Proposition 3.4. Suppose $\left(i_{1} j_{1} i_{2} j_{2} i_{1}\right)$ is a homotopically nontrivial closed path in $G^{\prime}$. Then

(i) $s_{i_{1} i_{2}}^{j_{1} j_{2}}$ acts as the identity on $F=X_{i_{1}} \cap X_{i_{2}}$;

(ii) The 2-dimensional subspace $\left\langle\mathbf{e}_{j_{1}}, \mathbf{e}_{j_{2}}\right\rangle$ is not contained in the hyperplane $X_{i_{1}}$, therefore $L=X_{i_{1}} \cap\left\langle\mathbf{e}_{j_{1}}, \mathbf{e}_{j_{2}}\right\rangle$ is a line; (iii) $s_{i_{1} i_{2}}^{j_{1} j_{2}}$ stretches the line $L$ by a factor of $c_{i_{1} i_{2}}^{j_{1} j_{2}}$.

Moreover, if $c_{i_{1} i_{2}}^{j_{1} j_{2}} \neq 1$, then $F$ is a codimension 1 subspace in $X_{i_{1}}$, and we have $X_{i_{1}}=F \oplus L$.

Proof. We first prove statement (i). The map $s_{i_{1} i_{2}}^{j_{1} j_{2}}$ is the composition of a projection from $X_{i_{2}}$ to $X_{i_{1}}$ and a projection from $X_{i_{1}}$ to $X_{i_{2}}$, both of which act on $X_{i_{1}} \cap X_{i_{2}}$ as the identity.

For part (ii), note that $j_{1} \neq j_{2}$, otherwise $\left(i_{1} j_{1} i_{2} j_{2} i_{1}\right)$ is contractible. Hence $\left\langle\mathbf{e}_{j_{1}}, \mathbf{e}_{j_{2}}\right\rangle$ is a 2-dimensional subspace. Since $\omega_{i_{1} j_{1}} \neq 0$ and $\omega_{i_{1} j_{2}} \neq 0$, 
the vectors $\mathbf{e}_{j_{1}}$ and $\mathbf{e}_{j_{2}}$ are not orthogonal to the row vector $\mathbf{e}_{i_{1}}^{T} \Omega$, hence they are not contained in $X_{i_{1}}$.

For part (iii), observe that the line $X_{i_{1}} \cap\left\langle\mathbf{e}_{j_{1}}, \mathbf{e}_{j_{2}}\right\rangle$ is generated by $\mathbf{v}=$ $\omega_{i_{1} j_{2}} \mathbf{e}_{j_{1}}-\omega_{i_{1} j_{1}} \mathbf{e}_{j_{2}}$. Since $s_{i_{2} \leftarrow j_{1}}$ is a projection on $X_{i_{2}}$ in the direction of $\mathbf{e}_{j_{1}}$, we have

$$
s_{i_{2} \leftarrow j_{1}}(\mathbf{v})=\frac{\omega_{i_{1} j_{1}}}{\omega_{i_{2} j_{1}}}\left(\omega_{i_{2} j_{2}} \mathbf{e}_{j_{1}}-\omega_{i_{2} j_{1}} \mathbf{e}_{j_{2}}\right) .
$$

Similarly,

$$
s_{i_{1} \leftarrow j_{2}}\left(s_{i_{2} \leftarrow j_{1}}(\mathbf{v})\right)=\frac{\omega_{i_{1} j_{1}} \omega_{i_{2} j_{2}}}{\omega_{i_{2} j_{1}} \omega_{i_{1} j_{2}}}\left(\omega_{i_{1} j_{2}} \mathbf{e}_{j_{1}}-\omega_{i_{1} j_{1}} \mathbf{e}_{j_{2}}\right)=c_{i_{1} i_{2}}^{j_{1} j_{2}} \mathbf{v} .
$$

Finally, the condition $c_{i_{1} i_{2}}^{j_{1} j_{2}} \neq 1$ implies that the rows of $\Omega_{i_{1} i_{2}}^{j_{1} j_{2}}$ are not constant multiples of each other, hence $X_{i_{1}} \neq X_{i_{2}}$. Since both $X_{i_{1}}$ and $X_{i_{2}}$ are hyperplanes in $V$, their intersection has codimension 1 in $X_{i_{1}}$.

In summary, the linear map $s_{\gamma}$ takes a very simple form when $\gamma=$ $\left(i_{1} j_{1} i_{2} j_{2} i_{1}\right)$ is a closed path with $c_{i_{1} i_{2}}^{j_{1} j_{2}} \neq 1$ : it fixes a hyperplane and stretches a line by the positive factor $c_{i_{1} i_{2}}^{j_{1} j_{2}}$. In the next section we consider these linear maps as building blocks for constructing more complicated linear maps.

3.4. Linear endomorphisms of planes. Let $W$ be a 2-dimensional vector space over $\mathbb{R}$. For any $\mathbf{v} \in W$ and $a>0$ denote by

- $\operatorname{Fix}(\mathbf{v}, a) \subset \mathrm{GL}^{+}(W)$ the group of linear maps fixing the vector $\mathbf{v}$ and having determinant $a^{n}$ for some integer $n$;

- $\operatorname{Fix}(\mathbf{v}) \subset \mathrm{GL}^{+}(W)$ the group of linear maps fixing the vector $\mathbf{v}$ and having positive determinant.

Lemma 3.5. Let $\mathbf{v} \in W$ and $f_{1}, f_{2} \in \operatorname{Fix}(\mathbf{v})$. Suppose that $f_{1}$ and $f_{2}$ have linearly independent eigenvectors $\mathbf{w}_{1}$ and $\mathbf{w}_{2}$ with eigenvalues $a_{1}>1$ and $a_{2}>1$, respectively. Then $\operatorname{Fix}\left(\mathbf{v}, a_{1}\right)$ and $\operatorname{Fix}\left(\mathbf{v}, a_{2}\right)$ are contained in the closure $\overline{\left\langle f_{1}, f_{2}\right\rangle} \subset \mathrm{GL}^{+}(W)$.

Proof. In the basis $\left(\mathbf{w}_{1}, \mathbf{v}\right)$ the maps $f_{1}$ and $f_{2}$ are described by the matrices

$$
A_{1}=\left(\begin{array}{cc}
a_{1} & 0 \\
0 & 1
\end{array}\right) \text { and } A_{2}=\left(\begin{array}{cc}
a_{2} & 0 \\
b & 1
\end{array}\right)
$$

where $b \neq 0$. Define

$$
C_{n}=A_{1}^{n} A_{2} A_{1}^{-n}=\left(\begin{array}{cc}
a_{2} & 0 \\
\frac{b}{a_{1}^{n}} & 1
\end{array}\right)
$$

and let

$$
C_{n+1} C_{n}^{-1}=\left(\begin{array}{cc}
1 & 0 \\
\frac{b\left(1-a_{1}\right)}{a_{1}^{n+1} a_{2}} & 1
\end{array}\right) .
$$


The bottom left entry of $C_{n+1} C_{n}^{-1}$ tends to zero, therefore powers of $C_{n+1} C_{n}^{-1}$ are dense in the subgroup of matrices of the form

$$
\left(\begin{array}{ll}
1 & 0 \\
* & 1
\end{array}\right)
$$

Finally, note that multiplying these matrices by powers of $A_{i}$ yields everything in

$$
\operatorname{Fix}\left(\mathbf{v}, a_{i}\right)=\left\{\left(\begin{array}{cc}
a_{i}^{n} & 0 \\
t & 1
\end{array}\right): n \in \mathbb{Z}, t \in \mathbb{R}\right\}
$$

for $i=1,2$.

Lemma 3.6. Let $\mathbf{v}_{1}, \mathbf{v}_{2} \in W$ be linearly independent vectors, and let $a_{1}, a_{2}>$ 0 such that $\left\langle a_{1}, a_{2}\right\rangle$ is dense in $\mathbb{R}_{+}^{\times}$. Then

$$
\overline{\left\langle\operatorname{Fix}\left(\mathbf{v}_{1}, a_{1}\right), \operatorname{Fix}\left(\mathbf{v}_{2}, a_{2}\right)\right\rangle}=\mathrm{GL}^{+}(W) .
$$

Proof. Under the action of $\operatorname{Fix}\left(\mathbf{v}_{1}, a_{1}\right)$, the orbit of any vector that is not a constant multiple of $\mathbf{v}_{1}$ is a collection of lines parallel to $\mathbf{v}_{1}$. A similar statement is true for $\operatorname{Fix}\left(\mathbf{v}_{2}, a_{2}\right)$. Since $\mathbf{v}_{1}$ and $\mathbf{v}_{2}$ are linearly independent, it follows that

$$
H=\left\langle\operatorname{Fix}\left(\mathbf{v}_{1}, a_{1}\right), \operatorname{Fix}\left(\mathbf{v}_{2}, a_{2}\right)\right\rangle
$$

acts transitively on nonzero vectors.

Conjugating $\operatorname{Fix}\left(\mathbf{v}_{1}, a_{1}\right)$ by an element of $H$ mapping $\mathbf{v}_{1}$ to $\mathbf{v}_{2}$ shows that $\operatorname{Fix}\left(\mathbf{v}_{2}, a_{1}\right) \subset H$. Since $\left\langle a_{1}, a_{2}\right\rangle$ is dense in $\mathbb{R}_{+}^{\times}$, we have

$$
\overline{\left\langle\operatorname{Fix}\left(\mathbf{v}_{2}, a_{1}\right), \operatorname{Fix}\left(\mathbf{v}_{2}, a_{2}\right)\right\rangle}=\operatorname{Fix}\left(\mathbf{v}_{2}\right)
$$

and $\operatorname{Fix}\left(\mathbf{v}_{2}\right) \subset \bar{H}$.

Finally, notice that any $f \in \mathrm{GL}^{+}(W)$ can be written as $f_{2} \circ f_{1}$ where $f_{1} \in H$ sends $\mathbf{v}_{2}$ to $f\left(\mathbf{v}_{2}\right)$ and $f_{2} \in \operatorname{Fix}\left(\mathbf{v}_{2}\right)$. Hence $\bar{H}=\mathrm{GL}^{+}(W)$.

3.5. Cross-ratio groups of matrices. The final ingredient for the proof of Theorem 3.2 is Lemma 3.10 below, which relates dense cross-ratio groups to the density of eigenvalues of the maps $f_{\gamma}$ in the complex plane.

Lemma 3.7. If $M$ is a $2 \times 3$ matrix and $M_{i}$ denotes its $2 \times 2$ submatrix obtained by deleting the ith column, then $\operatorname{cr}\left(M_{1}\right) \operatorname{cr}\left(M_{3}\right)=\operatorname{cr}\left(M_{2}\right)$.

Proof. $\operatorname{cr}\left(M_{1}\right) \operatorname{cr}\left(M_{3}\right)=\frac{m_{12} m_{23}}{m_{22} m_{13}} \cdot \frac{m_{11} m_{22}}{m_{12} m_{21}}=\frac{m_{11} m_{23}}{m_{13} m_{21}}=\operatorname{cr}\left(M_{2}\right)$.

Corollary 3.8. If $M$ is a $2 \times 3$ matrix with nontrivial cross-ratio group, then the cross-ratio of at least two of its $2 \times 2$ submatrices is not 1 .

Proposition 3.9. Let $M$ be a $3 \times 3$ matrix of full rank and dense cross-ratio group. Then $M$ has two $2 \times 2$ submatrices with the following two properties:

(i) they are not contained in the same two rows or the same two columns

(ii) their cross-ratios generate a dense subgroup of $\mathbb{R}_{+}^{\times}$. 
Proof. Since $M$ has dense cross-ratio group, there are two $2 \times 2$ submatrices $M_{1}$ and $M_{2}$ that satisfy (ii). If they also satisfy (i), then we are done, so assume for example that they are in the same two columns. Then we can replace $M_{2}$ by another $2 \times 2$ matrix in the same two rows so that (ii) is still satisfied; otherwise the other two $2 \times 2$ submatrices sharing the rows of $M_{2}$ would have cross-ratios that are rational powers of $\operatorname{cr}\left(M_{1}\right)$, and by Lemma 3.7 the same would be true for $\operatorname{cr}\left(M_{2}\right)$.

Lemma 3.10. Suppose that the upper left $6 \times 6$ submatrix of $\Omega$ has the form

$$
\left(\begin{array}{cc}
0 & Y \\
Y^{T} & 0
\end{array}\right)
$$

for a $3 \times 3$ matrix $Y$ with positive entries such that $\operatorname{rank}(Y)=3$ and $\mathrm{CRG}(Y)$ is dense in $\mathbb{R}_{+}^{\times}$.

Let $\varepsilon>0$ and $u(x)=x^{2}+a x+b \in \mathbb{R}[x]$ be arbitrary where $b>0$. Then there exists a closed path $\gamma$ in $\mathbf{G}(\Omega)$, and $v(x)=x^{2}+a^{\prime} x+b^{\prime} \in \mathbb{R}[x]$ with $\left|a-a^{\prime}\right|<\varepsilon$ and $\left|b-b^{\prime}\right|<\varepsilon$ such that $v(x) \mid \chi\left(f_{\gamma}\right)$.

Proof. We assume the notations of Sections 3.1 and 3.3 with $I=\{1,2,3\}$ and $J=\{4,5,6\}$. Note that $X_{1}$ is a 2-dimensional subspace in $V$. By Proposition 3.9, we may assume without loss of generality that $c_{12}^{45}$ and $c_{13}^{46}$ are not rational powers of each other.

For all $i \in\{2,3\}$ and $j, j^{\prime} \in\{4,5,6\}$ the map $s_{1 i}^{j j^{\prime}} \in \mathrm{GL}^{+}\left(X_{1}\right)$ acts as the identity on the line $F_{i}=X_{i} \cap X_{1}$ and stretches the line $L_{j j^{\prime}}=X_{1} \cap\left\langle\mathbf{e}_{j}, \mathbf{e}_{j^{\prime}}\right\rangle$ by $c_{1 i}^{j j^{\prime}}$ by Proposition 3.4. Note that the fact that $Y$ has full rank implies that $F_{2}$ and $F_{3}$ are distinct and $L_{45}, L_{56}$ and $L_{46}$ are pairwise distinct.

Let $H$ be the submonoid of $\mathrm{GL}^{+}\left(X_{1}\right)$ generated by the maps $s_{1 i}^{j j^{\prime}} \in$ $\mathrm{GL}^{+}\left(X_{1}\right)$ where $i \in\{2,3\}$ and $j, j^{\prime} \in\{4,5,6\}$. It is in fact a subgroup, since $s_{1 i}^{j j^{\prime}}$ and $s_{1 i}^{j^{\prime} j}$ are inverses of each other (cf. Section 3.3).

The maps $s_{12}^{45}, s_{12}^{56}$ and $s_{12}^{46}$ are elements of $\mathrm{GL}^{+}\left(X_{1}\right)$ fixing the line $F_{2}$ and stretching along the lines $L_{45}, L_{56}$ and $L_{46}$, respectively. Recall that the indices were chosen so that $c_{12}^{45} \neq 1$, so by Corollary 3.8, at least two of the stretch factors $c_{12}^{45}, c_{12}^{56}$ and $c_{12}^{46}$ are different from 1. By Lemma 3.5, $\operatorname{Fix}\left(F_{2}, c_{12}^{45}\right)$ is contained in $\bar{H}$. By applying the same reasoning for the maps $s_{13}^{45}, s_{13}^{56}$ and $s_{13}^{46}$, we get that $\operatorname{Fix}\left(F_{3}, c_{23}^{56}\right)$ is also contained in $\bar{H}$. Lemma 3.6 then implies $\bar{H}=\mathrm{GL}^{+}\left(X_{1}\right)$.

Now pick an element $g \in \mathrm{GL}^{+}\left(X_{1}\right)$ with $\chi(g)=u(x)$. Then pick an $h \in H$ sufficiently close to $g$ so that $\chi(h)=x^{2}+a^{\prime} x+b^{\prime}$ satisfies $\left|a-a^{\prime}\right|<\varepsilon$ and $\left|b-b^{\prime}\right|<\varepsilon$. By the definition of $H$, there is a closed path $\gamma$ with base point 1 visiting only the vertices $1,2,3$ and $4,5,6$ such that $h=s_{\gamma}$. So the statement follows by Proposition 3.3.

Proof of Theorem 3.2. Let $\Omega=i(C, C)$ and let $\theta \in \mathbb{C} \backslash\{0\}$ be arbitrary. Then $v(x)=(x-\theta)(x-\bar{\theta}) \in \mathbb{R}[x]$ has positive constant term, so by Lemma 3.10 there exist

- a sequence $\left(v_{j}(x)\right)_{j \in \mathbb{N}}$ of monic quadratic polynomials in $\mathbb{R}[x]$ and 
- a sequence $\left(\gamma_{j}\right)_{j \in \mathbb{N}}$ of closed paths in $\mathbf{G}(\Omega)$

such that

- $v_{j}(x) \rightarrow v(x)$ and

- $v_{j}(x) \mid \chi\left(f_{\gamma_{j}}\right)$ for every $j \in \mathbb{N}$.

Let $\left(\theta_{j}\right)_{j \in \mathbb{N}}$ be a sequence such that $v_{j}\left(\theta_{j}\right)=0$ for all $j \in \mathbb{N}$ and $\theta_{j} \rightarrow \theta$.

Assume for a moment that $\theta$ is not an algebraic unit of degree at most 2 , that is, $\theta$ is not a root of a polynomial $x^{2}+s x \pm 1$ for some $s \in \mathbb{Z}$. In this case, $\theta_{j}$ is not an algebraic unit if $j$ is large enough, because the set of algebraic units of degree at most 2 is a discrete subset of $\mathbb{C}$. Hence by Theorem 2.1, we have $\theta \in \overline{\mathcal{G P}(C)}$.

To complete the proof, note that the set of $\theta \in \mathbb{C}$ where $\theta \neq 0$ and $\theta$ is not an algebraic unit of degree at most 2 is dense in $\mathbb{C}$ so we have $\overline{\mathcal{G P}(C)}=\mathbb{C}$.

\section{Construction of Rich Collection of Curves}

In this section, we show that rich collections of curves exist on sufficiently complicated surfaces.

Proposition 4.1. If $\xi(S) \geq 3$, then there exists a rich collection of curves on $S$.

First we prove a lemma about intersection matrices. For a multicurve $B=\left\{b_{1}, \ldots, b_{\ell}\right\}$ on $S$ and a vector $\mathbf{s}=\left(s_{1}, \ldots, s_{\ell}\right)$ with integer coordinates, the product $T_{B}^{\mathrm{s}}=\prod_{j=1}^{\ell} T_{b_{j}}^{s_{j}}$ is called a multitwist about the multicurve $B$.

Lemma 4.2. Let $A$ and $B$ be multicurves of $S$. If $\mathbf{s}>0$ or $\mathbf{s}<0$, then

$$
i\left(A, T_{B}^{\mathbf{s}}(A)\right)=i(A, B) D_{|\mathbf{s}|} i(B, A)
$$

where $D_{|\mathbf{s}|}$ is the $\ell \times \ell$ diagonal matrix with entries $\left|s_{1}\right|, \ldots,\left|s_{\ell}\right|$ on the diagonal.

Proof. If $A, B$ and $C$ are multicurves on $S$ and $\mathbf{s}>0$ or $\mathbf{s}<0$, then we have

$$
\left|i\left(a, T_{B}^{\mathbf{s}}(c)\right)-\sum_{j=1}^{\ell}\right| s_{j}\left|i\left(a, b_{j}\right) i\left(b_{j}, c\right)\right| \leq i(a, c)
$$

for all $a \in A$ and $c \in C$ [FM12, Prop. 3.4]. We can summarize these inequalities in the single inequality

$$
\left|i\left(A, T_{B}^{\mathbf{s}}(C)\right)-i(A, B) D_{|\mathbf{s}|} i(B, C)\right| \leq i(A, C) .
$$

We obtain the claimed equation by setting $C=A$.

Proof of Proposition 4.1. Consider the pairs of multicurves $A, B$ on $S_{0,6}$, $S_{1,3}$ and $S_{2,0}$, pictured on Figure 4.1. In the three cases, the intersection 


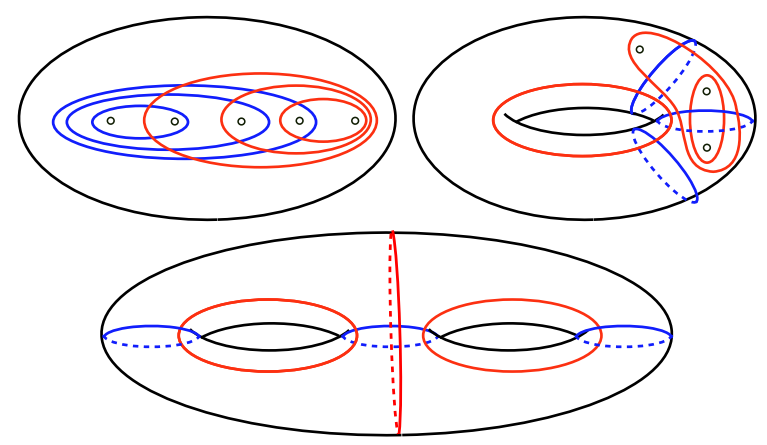

Figure 4.1. Curves

matrix $i(A, B)$ is

$$
\left(\begin{array}{lll}
2 & 2 & 2 \\
0 & 2 & 2 \\
0 & 0 & 2
\end{array}\right), \quad\left(\begin{array}{lll}
1 & 1 & 1 \\
0 & 2 & 2 \\
0 & 0 & 2
\end{array}\right) \text { and }\left(\begin{array}{lll}
1 & 1 & 0 \\
0 & 2 & 0 \\
0 & 1 & 1
\end{array}\right)
$$

respectively. In the first two cases $i(A, B) i(B, A)$ is

$$
\left(\begin{array}{ccc}
12 & 8 & 4 \\
8 & 8 & 4 \\
4 & 4 & 4
\end{array}\right) \text { and }\left(\begin{array}{lll}
3 & 4 & 2 \\
4 & 8 & 4 \\
2 & 4 & 4
\end{array}\right)
$$

In the third case

$$
i(A, B)\left(\begin{array}{lll}
1 & 0 & 0 \\
0 & 1 & 0 \\
0 & 0 & 2
\end{array}\right) i(B, A)=\left(\begin{array}{lll}
2 & 2 & 1 \\
2 & 4 & 2 \\
1 & 2 & 3
\end{array}\right) .
$$

By Lemma 4.2, we obtain a pair of multicurves on all three surfaces with rank 3 intersection matrix with positive entries and dense cross-ratio group. The curves necessarily fill the surface in each case, so we obtain a rich collection of curves.

Any other compact orientable surface with $\xi(S) \geq 3$ can be obtained from the three surfaces above by removing open disks and taking connected sums with tori. Hence we obtain a collection $C$ on all these surfaces that satisfy all properties of richness except the filling property. However, the filling property is easily achieved by extending both multicurves to maximal multicurves, being careful not to include the same curve in both maximal multicurves.

We are now ready to prove Theorem 1.2.

Theorem 1.2. If $S$ is a compact orientable surface with $\xi(S) \geq 3$, then there is a collection of curves $C$ on $S$ such that $\overline{\mathcal{G P}(C)}=\mathbb{C}$.

Proof. There exists a rich collection of curves by Proposition 4.1. By Theorem 3.2 , this implies that $\overline{\mathcal{G P}(C)}=\mathbb{C}$. 
Acknowledgements. We are grateful to Ursula Hamenstädt, Autumn Kent, Dan Margalit and the referees for their comments and help.

\section{REFERENCES}

[Fat92] Albert Fathi. Démonstration d'un théorème de Penner sur la composition des twists de Dehn. Bull. Soc. Math. France, 120(4):467-484, 1992.

[FM12] Benson Farb and Dan Margalit. A primer on mapping class groups, volume 49 of Princeton Mathematical Series. Princeton University Press, Princeton, NJ, 2012.

[Fri85] David Fried. Growth rate of surface homeomorphisms and flow equivalence. Ergodic Theory Dynam. Systems, 5(4):539-563, 1985.

[Ham14] Ursula Hamenstädt. Typical properties of periodic teichmueller geodesics. preprint, arxiv:1409.5978, 2014.

[Pen88] Robert C. Penner. A construction of pseudo-Anosov homeomorphisms. Trans. Amer. Math. Soc., 310(1):179-197, 1988.

[SS15] Hyunshik Shin and Balázs Strenner. Pseudo-Anosov mapping classes not arising from Penner's construction. Geom. Topol., 19(6):3645-3656, 2015.

[Str15] Balazs Strenner. Algebraic degrees and Galois conjugates of pseudo-Anosov stretch factors. ProQuest LLC, Ann Arbor, MI, 2015. Thesis (Ph.D.)-The University of Wisconsin - Madison.

[Str16] Balázs Strenner. Algebraic degrees of pseudo-Anosov stretch factors. Preprint, arXiv:1506.06412, 2016.

[Thu88] William P. Thurston. On the geometry and dynamics of diffeomorphisms of surfaces. Bull. Amer. Math. Soc. (N.S.), 19(2):417-431, 1988.

[Thu14] William Thurston. Entropy in dimension one. preprint, arXiv:1402.2008, 2014.

[Tio13] Giulio Tiozzo. Galois conjugates of entropies of real unimodal maps. preprint, arXiv:1310.7647, 2013.

School of Mathematics, Georgia Institute of Technology, 686 Cherry Street NW, Atlanta GA 30332-0160, USA

E-mail address: strenner@math.gatech.edu 